\title{
ON BOBKOV'S APPROXIMATE DE FINETTI REPRESENTATION VIA APPROXIMATION OF PERMANENTS OF COMPLEX RECTANGULAR MATRICES
}

\author{
BERO ROOS \\ (Communicated by Walter Craig)
}

\begin{abstract}
Bobkov (J. Theoret. Probab. 18(2) (2005) 399-412) investigated an approximate de Finetti representation for probability measures, on product measurable spaces, which are symmetric under permutations of coordinates. One of the main results of that paper was an explicit approximation bound for permanents of complex rectangular matrices, which was shown by a somewhat complicated induction argument. In this paper, we indicate how to avoid the induction argument using an (asymptotic) expansion. Our approach makes it possible to give new explicit higher order approximation bounds for such permanents and in turn for the probability measures mentioned above.
\end{abstract}

\section{INTRODUCTION}

Suppose that $X:=\left(X_{1}, X_{2}, X_{3}, \ldots\right)$ is an infinite exchangeable sequence of random variables on a probability space $(\Omega, \mathcal{A}, P)$ with values in a measurable space $(S, \mathcal{S})$, that is, the distribution $P^{X}$ of $X$ on the infinite product measurable space $\left(S^{\infty}, \mathcal{S}^{\otimes \infty}\right)$ is invariant under permutations of a finite number of coordinates. The de Finetti Theorem says that, under mild assumptions on the space $(S, \mathcal{S})$, there is a probability space $(T, \mathcal{T}, \nu)$ and a Markov kernel $\mu: T \times \mathcal{S} \longrightarrow[0,1],(t, A) \mapsto \mu_{t}(A)$ such that

$$
P^{X}=\int_{T}\left(\mu_{t}\right)^{\otimes \infty} \mathrm{d} \nu(t) .
$$

For instance, it suffices to assume that $(S, \mathcal{S})$ is a Borel (or standard) measurable space, i.e. Borel isomorphic to some Borel measurable subset of $\mathbb{R}$ (see Hewitt and Savage [10] or Diaconis and Freedman [6]).

For a finite exchangeable sequence, an analogous representation does not generally hold, but there are approximate de Finetti results. In what follows, let $N \in \mathbb{N}$, $n \in \underline{N}=\{1, \ldots, N\}$, and let $Y_{N}=\left(X_{1}, \ldots, X_{N}\right)$ be an exchangeable family of $S$ valued random variables, that is, the distribution $P^{Y_{N}}$ of $Y_{N}$, defined on $\left(S^{N}, \mathcal{S}^{\otimes N}\right)$, is invariant under permutation of coordinates. Let $Q_{1}$ be the probability measure on $\left(S^{n}, \mathcal{S}^{\otimes n}\right)$ defined by

$$
Q_{1}(A)=\int\left(\frac{1}{N} \sum_{j=1}^{N} \delta_{X_{j}(\omega)}\right)^{\otimes n}(A) \mathrm{d} P(\omega)
$$

Received by the editors March 13, 2012 and, in revised form, September 20, 2013.

2010 Mathematics Subject Classification. Primary 60G09, 62E17, 15A45.

Key words and phrases. de Finetti representation, permanent, Hadamard type inequality. 
for $A \in \mathcal{S}^{\otimes n}$, where $\delta_{x}$ denotes the Dirac measure at the point $x \in S$. In other words, $Q_{1}$ is the $P$-expectation of the $n$-th power of an empirical measure on $\left(S^{N}, \mathcal{S}^{\otimes N}\right)$. The following results can be found in Diaconis and Freedman [6]. They showed that

$$
d_{\mathrm{TV}}\left(P^{Y_{n}}, Q_{1}\right) \leq 1-\frac{N !}{(N-n) ! N^{n}} \leq \frac{n(n-1)}{2 N},
$$

where $d_{\mathrm{TV}}\left(R, R^{\prime}\right)=\sup _{A \in \mathcal{S} \otimes n}\left|R(A)-R^{\prime}(A)\right|$ denotes the total variation distance between finite signed measures $R$ and $R^{\prime}$ on $\left(S^{n}, \mathcal{S}^{\otimes n}\right)$. Hence, if $\frac{n^{2}}{N}$ is small, then $P^{Y_{n}}$ has an approximate de Finetti representation $Q_{1}$. It turned out that, in general, the bound (1.1) is sharp. However, if $S$ is finite and of cardinality $|S|=d \in \mathbb{N}$, then the nice inequality

$$
d_{\mathrm{TV}}\left(P^{Y_{n}}, Q_{1}\right) \leq \frac{d n}{N}
$$

is available, which, in the case of finite $S$, is better than (1.1) if $d$ is sufficiently small compared to $n$.

On the other hand, it is possible to obtain similar good bounds in the general case if the total variation distance is replaced by a weaker metric. Let $\mathcal{F}_{n}$ be the set of all functions $f: S^{n} \longrightarrow \mathbb{C}$ such that measurable $f_{1}, \ldots, f_{n}: S \longrightarrow \mathbb{C}$ exist with $\left|f_{k}\left(x_{k}\right)\right| \leq 1$ for $k \in \underline{n}$ and $f(x)=\prod_{k=1}^{n} f_{k}\left(x_{k}\right)$ for all $x=\left(x_{1}, \ldots, x_{n}\right) \in$ $S^{n}$. We write $f=\bigotimes_{k=1}^{n} f_{k}$. Furthermore, let $\underline{N}_{\neq}^{n}=\left\{\left(j_{1}, \ldots, j_{n}\right) \in \underline{N}^{n} \mid j_{k} \neq\right.$ $j_{\ell}$ for all $k, \ell \in \underline{n}$ with $\left.k \neq \ell\right\}$.

Bobkov [3] showed in his Theorem 1.1 (see also p. 405 there) the inequality

$$
\sup _{f \in \mathcal{F}_{n}}\left|\int f \mathrm{~d}\left(P^{Y_{n}}-Q_{1}\right)\right| \leq C \frac{n}{N} \quad \text { with } C=16 .
$$

For the proof, he used the representation

$$
\begin{aligned}
& \int f \mathrm{~d}\left(P^{Y_{n}}-Q_{1}\right)=\int\left(\prod_{k=1}^{n} f_{k}\left(X_{k}(\omega)\right)-\int f \mathrm{~d}\left(\frac{1}{N} \sum_{j=1}^{N} \delta_{X_{j}(\omega)}\right)^{\otimes n}\right) \mathrm{d} P(\omega) \\
& (1.4) \quad=\int\left(\frac{(N-n) !}{N !} \sum_{j \in \underline{N}_{\neq}^{n}} \prod_{k=1}^{n} f_{k}\left(X_{j_{k}}(\omega)\right)-\prod_{k=1}^{n}\left(\frac{1}{N} \sum_{j=1}^{N} f_{k}\left(X_{j}(\omega)\right)\right)\right) \mathrm{d} P(\omega)
\end{aligned}
$$

for $f=\bigotimes_{k=1}^{n} f_{k} \in \mathcal{F}_{n}$ and a remarkable approximation result for permanents of complex rectangular matrices (see Theorem A below), which he proved by using a somewhat complicated induction argument. The permanent of a complex rectangular matrix $Z=\left(z_{j, k}\right) \in \mathbb{C}^{N \times n}$ with $N \in \mathbb{N}$ and $n \in \underline{N}$ is defined by

$$
\operatorname{Per}(Z):=\sum_{j \in \underline{N}_{\neq}^{n}} \prod_{k=1}^{n} z_{j_{k}, k} .
$$

For general properties of permanents, we refer the reader to Minc [13] and Cheon and Wanless [5].

Theorem A (Bobkov [3, Theorem 2.1]). Let $N \in \mathbb{N}, n \in \underline{N}$ and $Z=\left(z_{j, k}\right) \in$ $\mathbb{C}^{N \times n}$. For $j \in \underline{N}$ and $k \in \underline{n}$, we assume that $\left|z_{j, k}\right| \leq 1$ and set $\widetilde{z}_{k}=\frac{1}{N} \sum_{j=1}^{N} z_{j, k}$. 
Then

$$
\left|\frac{(N-n) !}{N !} \operatorname{Per}(Z)-\prod_{k=1}^{n} \widetilde{z}_{k}\right| \leq C \frac{n}{N} \quad \text { with } C=16 .
$$

From Proposition 4.1 in Bobkov [2] it follows that (1.3) and (1.5) hold with the better constant $C=6$ if $z_{j, k}=z_{j, 1}$ for all $j \in \underline{N}$ and $k \in \underline{n}$. However, Theorem 2.13 below shows that $C$ can always be taken smaller than 3.57.

For two finite signed measures $R$ and $R^{\prime}$ on $\left(S^{n}, \mathcal{S}^{\otimes n}\right)$, let

$$
d_{\mathrm{PV}}\left(R, R^{\prime}\right)=\sup _{A_{1}, \ldots, A_{n} \in \mathcal{S}}\left|R\left(A_{1} \times \ldots \times A_{n}\right)-R^{\prime}\left(A_{1} \times \ldots \times A_{n}\right)\right|
$$

denote the so-called product variation between $R$ and $R^{\prime}$. Obviously $d_{\mathrm{PV}}$ is a metric on the set of all finite signed measures on $\left(S^{n}, \mathcal{S}^{\otimes n}\right)$. Furthermore,

$$
d_{\mathrm{PV}}\left(R, R^{\prime}\right) \leq \sup _{f \in \mathcal{F}_{n}}\left|\int f \mathrm{~d}\left(R-R^{\prime}\right)\right|
$$

Therefore (1.3) and the inequalities of Theorem 1.1 below imply bounds for $d_{\mathrm{PV}}$.

In the next section, we present refinements of (1.5); see Theorems 2.8, 2.13 and Corollary 2.12, The latter together with (1.4) and a similar representation implies Theorem 1.1 below, the first part of which is better than (1.3) with $C=3.57$ if $\frac{n}{N} \leq \frac{1}{2}$. The second part shows that, if $n \geq 2$ and in turn $N \geq 2$, a more accurate approximation of $P^{Y_{n}}$ by a finite signed measure $Q_{2}$ on $\left(S^{n}, \mathcal{S}^{\otimes n}\right)$ is possible, where

$$
Q_{2}(A)=Q_{1}(A)-\frac{1}{N(N-1)} \sum_{K \subseteq \underline{n}:|K|=2} \sum_{j=1}^{N} \int\left(\bigotimes_{k \in \underline{n}} R_{j, k, K}(\omega)\right)(A) \mathrm{d} P(\omega)
$$

for $A \in \mathcal{S}^{\otimes n}$ and

$$
R_{j, k, K}(\omega)= \begin{cases}\delta_{X_{j}(\omega)}-\frac{1}{N} \sum_{\ell=1}^{N} \delta_{X_{\ell}(\omega),} & \text { if } k \in K, \\ \frac{1}{N} \sum_{\ell=1}^{N} \delta_{X_{\ell}(\omega)}, & \text { if } k \in \underline{n} \backslash K .\end{cases}
$$

Theorem 1.1. Under the assumptions above and if $\frac{n}{N}<1$, we have

$$
\begin{aligned}
& \sup _{f \in \mathcal{F}_{n}}\left|\int f \mathrm{~d}\left(P^{Y_{n}}-Q_{1}\right)\right| \leq \frac{n}{N}+2.12 \frac{\left(\frac{n}{N}\right)^{3 / 2}}{\left(1-\frac{n}{N}\right)^{3 / 4}}, \\
& \sup _{f \in \mathcal{F}_{n}}\left|\int f \mathrm{~d}\left(P^{Y_{n}}-Q_{2}\right)\right| \leq \sqrt{3}\left(\frac{n}{N}\right)^{3 / 2}+2.27 \frac{\left(\frac{n}{N}\right)^{2}}{\left(1-\frac{n}{N}\right)^{3 / 4}}, \quad \text { if } n \geq 2 .
\end{aligned}
$$

Higher order results are also possible using Theorem 2.8 or Theorem 2.13 below. We omit the details.

\section{Approximation of permanents}

For $n \in \mathbb{N}$, the indeterminate $x=\left(x_{1}, \ldots, x_{n}\right)$ and $r \in \mathbb{Z}_{+}^{n}=\{0,1,2, \ldots\}^{n}$, we set $x^{r}=\prod_{k \in \underline{n}} x_{k}^{r_{k}}$ and write $a_{r}=\operatorname{Coeff}\left(x^{r} ; \sum_{s \in \mathbb{Z}_{+}^{n}} a_{s} x^{s}\right)$ for the coefficient of $x^{r}$ in the formal power series $\sum_{s \in \mathbb{Z}_{+}^{n}} a_{s} x^{s},\left(a_{s} \in \mathbb{C}\right)$. Sometimes $y$ will be 
our indeterminate. However, the symbols $x$ and $y$ may have other meanings as indicated below. In what follows, we use the simple fact that, for $N \in \mathbb{N}, n \in \underline{N}$ and $Z=\left(z_{j, k}\right) \in \mathbb{C}^{N \times n}$,

$$
\operatorname{Per}(Z)=\operatorname{Coeff}\left(x_{1} \cdots x_{n} ; \prod_{j=1}^{N}\left(1+\sum_{k=1}^{n} z_{j, k} x_{k}\right)\right) .
$$

Furthermore, if additionally $Z$ has identical columns, i.e. $z_{j, k}=z_{j, 1}$ for all $j \in \underline{N}$ and $k \in \underline{n}$, then

$$
\operatorname{Per}(Z)=n ! \operatorname{Coeff}\left(y^{n} ; \prod_{j=1}^{N}\left(1+z_{j, 1} y\right)\right) .
$$

The main result of this section is Theorem 2.8 below and requires the following lemmas, the first of which plays a prominent role in the theory of polynomials over infinite dimensional spaces. Its proof is due to Hörmander [11, Theorem 4]; see also Harris [9] and Dineen [7, Proposition 1.44 and the notes on page 79]. However, first versions for real spaces were already shown in Kellogg [12] and Banach [1].

Lemma 2.1. Let $n \in \mathbb{N}, E$ be a complex Hilbert space, $F$ be a complex Banach space, $g: E^{n} \longrightarrow F$ be n-linear (i.e. linear in each component), continuous and symmetric in its arguments. Let $\widehat{g}(x)=g(x, \ldots, x)$ for $x \in E$ and

$$
\begin{aligned}
& \|g\|=\sup \left\{\left\|g\left(x_{1}, \ldots, x_{n}\right)\right\| \mid x_{i} \in E,\left\|x_{i}\right\| \leq 1 \text { for each } i \in \underline{n}\right\} \\
& \|\widehat{g}\|=\sup \{\|\widehat{g}(x)\| \mid x \in E,\|x\| \leq 1\} .
\end{aligned}
$$

Then $\|g\|=\|\widehat{g}\|$.

The proof of the next lemma uses Lemma 2.1 and the Cauchy integral formula. We note that the more complicated Lemma 3 in [16] only yields a weaker result under the assumptions used here. We always set $0^{0}=1$.

Lemma 2.2. Let $N \in \mathbb{N}, n \in \underline{N}$ and $A=\left(a_{j, k}\right) \in \mathbb{C}^{N \times n}$. For each $k \in \underline{n}$, we assume that $\sum_{j=1}^{N} a_{j, k}=0$ and set $\alpha_{k}=\frac{1}{N} \sum_{j=1}^{N}\left|a_{j, k}\right|^{2}$. Then we have

$$
|\operatorname{Per}(A)| \leq \frac{n ! N^{N / 2}}{(N-n)^{(N-n) / 2} n^{n / 2}} \prod_{k=1}^{n} \sqrt{\alpha_{k}} .
$$

Proof. We may assume that $\alpha_{k} \neq 0$ for each $k \in \underline{n}$. Let $E=\left\{x={ }^{\mathrm{t}}\left(x_{1}, \ldots, x_{N}\right) \in\right.$ $\left.\mathbb{C}^{N \times 1} \mid \sum_{j=1}^{N} x_{j}=0\right\}$ be equipped with the standard inner product and consider $F=\mathbb{C}, g: E^{n} \longrightarrow F, g\left(x^{(1)}, \ldots, x^{(n)}\right)=\operatorname{Per}\left(x^{(1)}, \ldots, x^{(n)}\right)$ for $x^{(1)}, \ldots, x^{(n)} \in E$, where "t" denotes transposition. It is easily seen that Lemma 2.1 can be applied, which gives $|\operatorname{Per}(A)| \leq\|\widehat{g}\| N^{n / 2} \prod_{k=1}^{n} \sqrt{\alpha_{k}}$. Using (2.2), we obtain for $x \in E$ with $\|x\| \leq 1$ and arbitrary $r \in(0, \infty)$ that

$$
\begin{aligned}
|\widehat{g}(x)| & =\frac{n !}{2 \pi r^{n}}\left|\int_{-\pi}^{\pi} \mathrm{e}^{-\mathrm{i} n t} \prod_{j=1}^{N}\left(1+x_{j} r \mathrm{e}^{\mathrm{i} t}\right) \mathrm{d} t\right| \\
& \leq \frac{n !}{r^{n}} \sup _{t \in[-\pi, \pi]} \prod_{j=1}^{N}\left|1+x_{j} r \mathrm{e}^{\mathrm{i} t}\right| \leq \frac{n !}{r^{n}}\left(1+\frac{r^{2}}{N}\right)^{N / 2}
\end{aligned}
$$


the last inequality follows from the inequality between arithmetic and geometric means. Indeed, for $w \in E$, we have

$$
\prod_{j=1}^{N}\left|1+w_{j}\right|=\left(\prod_{j=1}^{N}\left(1+2 \operatorname{Re}\left(w_{j}\right)+\left|w_{j}\right|^{2}\right)\right)^{1 / 2} \leq\left(1+\frac{1}{N} \sum_{j=1}^{N}\left|w_{j}\right|^{2}\right)^{N / 2},
$$

where $\operatorname{Re}\left(w_{j}\right)$ denotes the real part of $w_{j}$. Let $\varepsilon \in(0, \infty)$ and

$$
r=\left(\frac{n N}{N-n+\varepsilon}\right)^{1 / 2}
$$

Letting $\varepsilon \rightarrow 0$ yields $\|\widehat{g}\| \leq \frac{n ! N^{(N-n) / 2}}{(N-n)^{(N-n) / 2} n^{n / 2}}$ and the result is shown.

Remark 2.3. Inequality (2.3) can be viewed as a Hadamard type inequality for permanents of matrices with zero column sums. Another inequality of this type is

$$
|\operatorname{Per}(Z)| \leq N ! \prod_{k=1}^{N}\left(\frac{1}{N} \sum_{j=1}^{N}\left|z_{j, k}\right|^{2}\right)^{1 / 2},
$$

which is valid for general quadratic matrices $Z=\left(z_{j, k}\right) \in \mathbb{C}^{N \times N}$ with $N \in \mathbb{N}$. Carlen et al. 4 gave two proofs of (2.4), which, however, also follows directly from Lemma 2.1 together with the inequality between arithmetic and geometric means.

Inequality (2.4) can be used to derive an alternative bound for the left-hand side of (2.3) as follows. Consider the assumptions of Lemma 2.2 and define $Z=\left(z_{j, k}\right) \in$ $\mathbb{C}^{N \times N}$ with $z_{j, k}=a_{j, k}$ for $j \in \underline{N}, k \in \underline{n}$ and $z_{j, k}=1$ for $j \in \underline{N}, k \in \underline{N} \backslash \underline{n}$. Then

$$
|\operatorname{Per}(A)|=\frac{|\operatorname{Per}(Z)|}{(N-n) !} \leq \frac{N !}{(N-n) !} \prod_{k=1}^{n} \sqrt{\alpha_{k}} .
$$

However, it turns out that (2.3) is always better than the inequality in (2.5), since

$$
\frac{N^{N}}{(N-n)^{N-n} n^{n}} \leq\left(\prod_{m=1}^{N-n} \frac{N-m+1}{N-n-m+1}\right)\left(\prod_{m=1}^{n} \frac{N-m+1}{n-m+1}\right)=\left(\begin{array}{c}
N \\
n
\end{array}\right)^{2} .
$$

Lemma 2.4. Let $n \in \mathbb{N}, m \in \underline{n}_{0}=\{0, \ldots, n\}$ and $w_{1, k}, w_{2, k} \in \mathbb{C}$ for $k \in \underline{n}$. Then

$$
\left|\operatorname{Coeff}\left(y^{m} ; \prod_{k=1}^{n}\left(w_{1, k}+w_{2, k} y\right)\right)\right| \leq\left(\begin{array}{c}
n \\
m
\end{array}\right)\left(\frac{1}{n} \sum_{k=1}^{n}\left|w_{2, k}\right|^{2}\right)^{m / 2}\left(\frac{1}{n} \sum_{k=1}^{n}\left|w_{1, k}\right|^{2}\right)^{(n-m) / 2} \text {. }
$$

Proof. Using Cauchy's inequality, we obtain

$$
\begin{aligned}
& \left|\operatorname{Coeff}\left(y^{m} ; \prod_{k=1}^{n}\left(w_{1, k}+w_{2, k} y\right)\right)\right|=\left|\sum_{K \subseteq \underline{n}:|K|=m}\left(\prod_{k \in K} w_{2, k}\right) \prod_{k \in \underline{n} \backslash K} w_{1, k}\right| \\
& \leq\left(\sum_{K \subseteq \underline{n}:|K|=m} \prod_{k \in K}\left|w_{2, k}\right|^{2}\right)^{1 / 2}\left(\sum_{K \subseteq \underline{n}:|K|=m} \prod_{k \in \underline{n} \backslash K}\left|w_{1, k}\right|^{2}\right)^{1 / 2} \\
& =\operatorname{Coeff}\left(y^{m} ; \prod_{k=1}^{n}\left(1+\left|w_{2, k}\right|^{2} y\right)\right)^{1 / 2} \operatorname{Coeff}\left(y^{n-m} ; \prod_{k=1}^{n}\left(1+\left|w_{1, k}\right|^{2} y\right)\right)^{1 / 2} .
\end{aligned}
$$

The assertion now follows from a result due to Maclaurin, which says that if $g_{1}, \ldots, g_{n} \in[0, \infty)$, then $\left(\frac{1}{\left(\begin{array}{c}n \\ \ell\end{array}\right)} \operatorname{Coeff}\left(y^{\ell} ; \prod_{k=1}^{n}\left(1+g_{k} y\right)\right)\right)^{1 / \ell}$ is non-increasing in $\ell \in \underline{n}$; see Hardy et al. [8, Theorem 52, page 52]. 
Lemma 2.5. Let $n, N \in \mathbb{N}, m \in \mathbb{Z}_{+}$with $m \leq \min \{n, N\},\left(a_{j, k}\right) \in \mathbb{C}^{N \times n}$ with $\sum_{j=1}^{N} a_{j, k}=0$ for all $k \in \underline{n}, b \in \mathbb{C}^{n}, \alpha=\frac{1}{n N} \sum_{j=1}^{N} \sum_{k=1}^{n}\left|a_{j, k}\right|^{2}, \beta=\frac{1}{n} \sum_{k=1}^{n}\left|b_{k}\right|^{2}$. Then

$$
\left|\operatorname{Coeff}\left(x_{1} \cdots x_{n} ;\left(\sum_{k=1}^{n} b_{k} x_{k}\right)^{n-m} \prod_{j=1}^{N}\left(1+\sum_{k=1}^{n} a_{j, k} x_{k}\right)\right)\right| \leq \frac{n ! N^{N / 2} \alpha^{m / 2} \beta^{(n-m) / 2}}{(N-m)^{(N-m) / 2} m^{m / 2}} .
$$

Proof. Let $\alpha_{k}=\frac{1}{N} \sum_{j=1}^{N}\left|a_{j, k}\right|^{2},(k \in \underline{n})$. An application of Lemma 2.2 gives

$$
\begin{aligned}
& \frac{1}{(n-m) !}\left|\operatorname{Coeff}\left(x_{1} \cdots x_{n} ;\left(\sum_{k=1}^{n} b_{k} x_{k}\right)^{n-m} \prod_{j=1}^{N}\left(1+\sum_{k=1}^{n} a_{j, k} x_{k}\right)\right)\right| \\
& =\left|\sum_{K \subseteq \underline{n}:|K|=n-m} \operatorname{Coeff}\left(x_{1} \cdots x_{n} ;\left(\prod_{k \in K}\left(b_{k} x_{k}\right)\right) \prod_{j=1}^{N}\left(1+\sum_{k=1}^{n} a_{j, k} x_{k}\right)\right)\right| \\
& =\left|\sum_{K \subseteq \underline{n}:|K|=n-m} \operatorname{Coeff}\left(\prod_{k \in \underline{n} \backslash K} x_{k} ; \prod_{j=1}^{N}\left(1+\sum_{k \in \underline{n} \backslash K} a_{j, k} x_{k}\right)\right) \prod_{k \in K} b_{k}\right| \\
& \leq \frac{m ! N^{N / 2}}{(N-m)^{(N-m) / 2} m^{m / 2}} \sum_{K \subseteq \underline{n}:|K|=n-m}\left(\prod_{k \in \underline{n} \backslash K} \sqrt{\alpha_{k}}\right) \prod_{k \in K}\left|b_{k}\right| \\
& =\frac{m ! N^{N / 2}}{(N-m)^{(N-m) / 2} m^{m / 2}} \operatorname{Coeff}\left(y^{m} ; \prod_{k=1}^{n}\left(\left|b_{k}\right|+\sqrt{\alpha_{k}} y\right)\right) .
\end{aligned}
$$

The proof is easily completed using Lemma 2.4.

Lemma 2.6. For $r \in \mathbb{Z}_{+}, t, x \in[0,1]$, we have $\sum_{m=0}^{r}(m+1)^{t} x^{m} \leq\left(\frac{1-x^{r+1}}{1-x}\right)^{1+t}$.

Proof. This follows from $\sum_{m=0}^{r}(m+1) x^{m}=\frac{1-(r+2) x^{r+1}+(r+1) x^{r+2}}{(1-x)^{2}} \leq\left(\frac{1-x^{r+1}}{1-x}\right)^{2}$ and Hölder's inequality, i.e. $\sum_{m=0}^{r}(m+1)^{t} x^{m} \leq\left(\sum_{m=0}^{r}(m+1) x^{m}\right)^{t}\left(\sum_{m=0}^{r} x^{m}\right)^{1-t}$.

The following lemma is more precise than Lemma 3 in [15].

Lemma 2.7. Let $\ell, m, N \in \mathbb{N}, \ell \leq m \leq N$ and $C_{\ell}=\left(\frac{\mathrm{e}^{\ell} \ell !}{\ell^{\ell+1 / 2}}\right)^{1 / 2}$. Then

$$
\frac{N^{N / 2}}{(N-m)^{(N-m) / 2} m^{m / 2+1 / 4}\left(\begin{array}{l}
N \\
m
\end{array}\right)^{1 / 2}} \leq C_{\ell} .
$$

Proof. Let $p(m, N)=\frac{N^{N}}{(N-m)^{N-m}\left(\begin{array}{c}N \\ m\end{array}\right)}$. Since $q(k):=\left(\frac{k}{k+1}\right)^{k}$ is decreasing in $k \in \mathbb{Z}_{+}$, we have

$$
\frac{p(m, N)}{p(m, N+1)}=\frac{N^{N}(N+1-m)^{N+1-m}(N+1)}{(N+1)^{N+1}(N-m)^{N-m}(N+1-m)}=\frac{q(N)}{q(N-m)} \leq 1 .
$$

Hence $p(m, N) \leq \lim _{\widetilde{N} \rightarrow \infty} p(m, \tilde{N})=\mathrm{e}^{m} m$ ! and therefore the left-hand side of (2.6) is bounded by $\left(\frac{\mathrm{e}^{m} m !}{m^{m+1 / 2}}\right)^{1 / 2}$. Since this is decreasing in $m$ (cf. Mitrinović [14, p. 183]), the assertion follows.

We now present our first main result, which generalizes Theorem A. Indeed, it will turn out that $\gamma \leq \frac{n}{N}$ and, for $\ell=1, H_{\ell}(Z)=\prod_{k=1}^{n} \widetilde{z}_{k}$; see Remarks 2.9 and 2.10 below. A further advantage of $\gamma$ is that it can be equal to zero, namely in the case $z_{j, k}=\widetilde{z}_{k}$ for all $j \in \underline{N}$ and $k \in \underline{n}$. We note that the singularity in (2.7) can be removed; see Theorem 2.13 below. 
Theorem 2.8. Let $N \in \mathbb{N}, n \in \underline{N}, \ell \in \underline{n}$ and $Z=\left(z_{j, k}\right) \in \mathbb{C}^{N \times n}$. For $j \in \underline{N}$ and $k \in \underline{n}$, we assume that $\left|z_{j, k}\right| \leq 1$ and set $\widetilde{z}_{k}=\frac{1}{N} \sum_{j=1}^{N} z_{j, k}, a_{j, k}=z_{j, k}-\widetilde{z}_{k}$, $U_{j}(x)=\sum_{k=1}^{n} a_{j, k} x_{k}$, where $x=\left(x_{1}, \ldots, x_{n}\right)$ is an indeterminate. Further, let $C_{\ell}$ be as in Lemma 2.7.

$$
\begin{aligned}
\alpha & =\frac{1}{n N} \sum_{j=1}^{N} \sum_{k=1}^{n}\left|a_{j, k}\right|^{2}, \quad \beta=\frac{1}{n} \sum_{k=1}^{n}\left|\widetilde{z}_{k}\right|^{2}, \quad \gamma=\frac{n \alpha}{N} \min \left\{n, \frac{1}{1-\beta}\right\}, \\
G_{m}(Z) & =\frac{(N-m) !}{(n-m) ! N !} \operatorname{Coeff}\left(x_{1} \cdots x_{n} ;\left(\prod_{j=1}^{N}\left(1+U_{j}(x)\right)\right)\left(\sum_{k=1}^{n} \widetilde{z}_{k} x_{k}\right)^{n-m}\right)
\end{aligned}
$$

for $m \in \underline{n}_{0}$ and set $H_{\ell}(Z)=\sum_{m=0}^{\ell} G_{m}(Z)$. If $\gamma<1$, then

$$
\left|\frac{(N-n) !}{N !} \operatorname{Per}(Z)-H_{\ell}(Z)\right| \leq(\ell+1)^{1 / 4} C_{\ell+1} \frac{\gamma^{(\ell+1) / 2}}{(1-\gamma)^{3 / 4}} .
$$

Proof. Let $W_{m}(x)=\operatorname{Coeff}\left(y^{m} ; \prod_{j=1}^{N}\left(1+U_{j}(x) y\right)\right)$ for $m \in \underline{N}_{0}$. In view of (2.1),

$$
\begin{aligned}
& \prod_{j=1}^{N}\left(1+\sum_{k=1}^{n} z_{j, k} x_{k}\right)=\prod_{j=1}^{N}\left(U_{j}(x)+1+\sum_{k=1}^{n} \widetilde{z}_{k} x_{k}\right) \\
& =\sum_{m=0}^{N} W_{m}(x)\left(1+\sum_{k=1}^{n} \widetilde{z}_{k} x_{k}\right)^{N-m}=\sum_{m=0}^{N} \sum_{r=0}^{N-m}\left(\begin{array}{c}
N-m \\
r
\end{array}\right) W_{m}(x)\left(\sum_{k=1}^{n} \widetilde{z}_{k} x_{k}\right)^{r},
\end{aligned}
$$

and

$$
G_{m}(Z)=\frac{(N-m) !}{(n-m) ! N !} \operatorname{Coeff}\left(x_{1} \cdots x_{n} ; W_{m}(x)\left(\sum_{k=1}^{n} \widetilde{z}_{k} x_{k}\right)^{n-m}\right),
$$

we see that

$$
\begin{aligned}
\operatorname{Per}(Z) & =\sum_{m=0}^{n}\left(\begin{array}{c}
N-m \\
n-m
\end{array}\right) \operatorname{Coeff}\left(x_{1} \cdots x_{n} ; W_{m}(x)\left(\sum_{k=1}^{n} \widetilde{z}_{k} x_{k}\right)^{n-m}\right) \\
& =\frac{N !}{(N-n) !} \sum_{m=0}^{n} G_{m}(Z)
\end{aligned}
$$

and therefore $\frac{(N-n) !}{N !} \operatorname{Per}(Z)=H_{n}(Z)$. Using Lemmas 2.5 and 2.7 and the simple inequality $\left(\begin{array}{c}n \\ m\end{array}\right) \leq\left(\begin{array}{c}N \\ m\end{array}\right)\left(\frac{n}{N}\right)^{m}$ for $m \in \underline{n}_{0}$, we obtain

$$
\begin{aligned}
& \left|\frac{(N-n) !}{N !} \operatorname{Per}(Z)-H_{\ell}(Z)\right| \leq \sum_{m=\ell+1}^{n}\left|G_{m}(Z)\right| \\
& \leq \sum_{m=\ell+1}^{n} \frac{N^{N / 2}}{(N-m)^{(N-m) / 2} m^{m / 2}} \frac{\left(\begin{array}{c}
n \\
m
\end{array}\right)}{\left(\begin{array}{c}
N \\
m
\end{array}\right)} \alpha^{m / 2} \beta^{(n-m) / 2} \\
& \leq C_{\ell+1} \sum_{m=\ell+1}^{n} \frac{\left(\begin{array}{c}
n \\
m
\end{array}\right)}{\left(\begin{array}{l}
N \\
m
\end{array}\right)^{1 / 2}} m^{1 / 4} \alpha^{m / 2} \beta^{(n-m) / 2} \\
& \leq C_{\ell+1} \sum_{m=\ell+1}^{n} m^{1 / 4} \gamma^{m / 2}\left(\left(\begin{array}{c}
n \\
m
\end{array}\right) \max \left\{1-\beta, \frac{1}{n}\right\}^{m} \beta^{n-m}\right)^{1 / 2},
\end{aligned}
$$


where we used that $\beta \in[0,1]$. By applying Cauchy's inequality and the fact that, since $\ell \geq 1, \sum_{m=\ell+1}^{n}\left(\begin{array}{c}n \\ m\end{array}\right) \frac{1}{n^{m}} \leq\left(1+\frac{1}{n}\right)^{n}-2 \leq \mathrm{e}-2<1$, we obtain

$$
\begin{aligned}
\left|\frac{(N-n) !}{N !} \operatorname{Per}(Z)-H_{\ell}(Z)\right| & \leq C_{\ell+1}\left(\sum_{m=\ell+1}^{n} \sqrt{m} \gamma^{m}\right)^{1 / 2} \\
& \leq(\ell+1)^{1 / 4} C_{\ell+1} \gamma^{(\ell+1) / 2}\left(\sum_{m=0}^{n-\ell-1} \sqrt{m+1} \gamma^{m}\right)^{1 / 2} .
\end{aligned}
$$

It remains to use Lemma 2.6 with $t=\frac{1}{2}$.

For the rest of the paper, let the notation of Theorem 2.8 hold.

Remark 2.9. We have $\gamma \leq \frac{n}{N}$, since

$$
\alpha=\frac{1}{n N} \sum_{j=1}^{N} \sum_{k=1}^{n}\left|z_{j, k}\right|^{2}-\beta \leq 1-\beta .
$$

In particular, if $\left|z_{j, k}\right|=1$ for all $j \in \underline{N}$ and $k \in \underline{n}$, then $\alpha=1-\beta$. Indeed, writing $z_{j, k}=u_{j, k}+\mathrm{i} v_{j, k}$ and $\widetilde{z}_{k}=\widetilde{u}_{k}+\mathrm{i} \widetilde{v}_{k}$ with $u_{j, k}, v_{j, k} \in \mathbb{R}, \widetilde{u}_{k}=\frac{1}{N} \sum_{j=1}^{N} u_{j, k}$ and $\widetilde{v}_{k}=\frac{1}{N} \sum_{j=1}^{N} v_{j, k}$, we obtain

$$
\begin{aligned}
\alpha & =\frac{1}{n N} \sum_{j=1}^{N} \sum_{k=1}^{n}\left(\left(u_{j, k}-\widetilde{u}_{k}\right)^{2}+\left(v_{j, k}-\widetilde{v}_{k}\right)^{2}\right) \\
& =\frac{1}{n N} \sum_{k=1}^{n}\left(\sum_{j=1}^{N}\left(u_{j, k}^{2}+v_{j, k}^{2}\right)-N\left(\widetilde{u}_{k}^{2}+\widetilde{v}_{k}^{2}\right)\right),
\end{aligned}
$$

from which (2.9) follows.

Let us now collect some properties of the first few $G_{m}(Z)$, where we always assume that $m \in \underline{n}_{0}$.

Remark 2.10. The first few $G_{m}(Z)$ can be evaluated as follows:

$$
\begin{aligned}
& G_{0}(Z)=\prod_{k=1}^{n} \widetilde{z}_{k}, \quad G_{1}(Z)=0, \\
& G_{2}(Z)=-\frac{(N-2) !}{N !} \sum_{K \subseteq \underline{n}:|K|=2}\left(\sum_{j=1}^{N} \prod_{k \in K} a_{j, k}\right) \prod_{k \in \underline{n} \backslash K} \widetilde{z}_{k}, \\
& G_{3}(Z)=2 \frac{(N-3) !}{N !} \sum_{K \subseteq \underline{n}:|K|=3}\left(\sum_{j=1}^{N} \prod_{k \in K} a_{j, k}\right) \prod_{k \in \underline{n} \backslash K} \widetilde{z}_{k} .
\end{aligned}
$$

In order to prove this, let

$$
V_{m}(x)=\sum_{j=1}^{N}\left(-U_{j}(x)\right)^{m}, \quad W_{m}(x)=\operatorname{Coeff}\left(y^{m} ; \prod_{j=1}^{N}\left(1+U_{j}(x) y\right)\right)
$$

for $m \in \underline{N}_{0}$. We have

$$
W_{m}(x)=-\frac{1}{m} \sum_{k=0}^{m-2} W_{k}(x) V_{m-k}(x) \quad \text { for } m \in \underline{N},
$$


which can be shown in the same way as (10) in [15]. In particular,

$$
\begin{aligned}
& W_{0}(x)=1, \quad W_{1}(x)=0, \quad W_{2}(x)=-\frac{1}{2} V_{2}(x), \\
& W_{3}(x)=-\frac{1}{3} V_{3}(x), \quad W_{4}(x)=\frac{1}{8}\left(V_{2}(x)\right)^{2}-\frac{1}{4} V_{4}(x) .
\end{aligned}
$$

In view of (2.8), (2.11) and

$$
\begin{aligned}
& \operatorname{Coeff}\left(x_{1} \cdots x_{n} ; V_{m}(x)\left(\sum_{k=1}^{n} \widetilde{z}_{k} x_{k}\right)^{n-m}\right) \\
& =(-1)^{m} \sum_{j=1}^{N} \operatorname{Coeff}\left(x_{1} \cdots x_{n} ;\left(U_{j}(x)\right)^{m}\left(\sum_{k=1}^{n} \widetilde{z}_{k} x_{k}\right)^{n-m}\right) \\
& =(-1)^{m}(n-m) ! m ! \sum_{j=1}^{N} \sum_{K \subseteq \underline{n}:|K|=m}\left(\prod_{k \in K} a_{j, k}\right) \prod_{k \in \underline{n} \backslash K} \widetilde{z}_{k},
\end{aligned}
$$

for $m \in \underline{n}$, we see that (2.10) is true. We note that the representations in (2.10) of $G_{2}(Z)$ and $G_{3}(Z)$ have a simple form, but the omitted ones of $G_{m}(Z)$ with $m \geq 4$ are more complicated.

From the above, we obtain that $H_{1}(Z)=\prod_{k=1}^{n} \widetilde{z}_{k}$ and, if $n \geq 2$,

$$
H_{2}(Z)=\prod_{k=1}^{n} \widetilde{z}_{k}-\frac{1}{N(N-1)} \sum_{K \subseteq \underline{n}:|K|=2}\left(\sum_{j=1}^{N} \prod_{k \in K} a_{j, k}\right) \prod_{k \in \underline{n} \backslash K} \widetilde{z}_{k} .
$$

Remark 2.11. Let us derive some bounds for $\left|G_{2}(Z)\right|$ and $\left|G_{3}(Z)\right|$. From (2.12) and Lemma 2.4 it follows that, for $m \in \underline{n}$,

$$
\begin{aligned}
& \left|\operatorname{Coeff}\left(x_{1} \cdots x_{n} ; V_{m}(x)\left(\sum_{k=1}^{n} \widetilde{z}_{k} x_{k}\right)^{n-m}\right)\right| \\
& \leq(n-m) ! m ! \sum_{j=1}^{N}\left|\operatorname{Coeff}\left(y^{m} ; \prod_{k=1}^{n}\left(\widetilde{z}_{k}+a_{j, k} y\right)\right)\right| \\
& \leq n ! \sum_{j=1}^{N}\left(\frac{1}{n} \sum_{k=1}^{n}\left|a_{j, k}\right|^{2}\right)^{m / 2} \beta^{(n-m) / 2},
\end{aligned}
$$

which together with (2.10) gives

$$
\begin{aligned}
\left|G_{2}(Z)\right| & \leq \frac{n(n-1)}{2(N-1)} \alpha \beta^{(n-2) / 2}, \\
\left|G_{3}(Z)\right| & \leq \frac{1}{3} \frac{n !(N-3) !}{(n-3) ! N !} \sum_{j=1}^{N}\left(\frac{1}{n} \sum_{k=1}^{n}\left|a_{j, k}\right|^{2}\right)^{3 / 2} \beta^{(n-3) / 2} .
\end{aligned}
$$

The inequalities given above can be used to derive bounds for $\left|G_{2}(Z)\right|$ and $\left|G_{3}(Z)\right|$ depending on $\gamma$. For precise calculations, we use the notation

$$
\gamma(d)=\frac{n \alpha}{N} \min \left\{d n, \frac{1}{1-\beta}\right\}
$$


for $d \in(0, \infty)$, giving $\gamma=\gamma(1)$. We have

$$
\begin{aligned}
\left|G_{2}(Z)\right| \leq & \gamma(1 / 2) \max \left\{\beta^{(n-2) / 2}, \frac{1}{2} n(1-\beta) \beta^{(n-2) / 2}\right\} \frac{N(n-1)}{(N-1) n} \leq \gamma(1 / 2) \\
\left|G_{3}(Z)\right| \leq & \sqrt{3} \frac{(n-1)(n-2) N^{2}}{(N-1)(N-2) n^{2}} \sum_{j=1}^{N}\left(\frac{1}{N^{2}} \sum_{k=1}^{n}\left|a_{j, k}\right|^{2} \min \left\{\frac{n}{3}, \frac{1}{1-\beta}\right\}\right)^{3 / 2} \\
& \times \max \left\{\beta^{(n-3) / 2},\left(\frac{n}{3}\right)^{3 / 2}(1-\beta)^{3 / 2} \beta^{(n-3) / 2}\right\} \\
\leq & \sqrt{3} \sum_{j=1}^{N}\left(\frac{1}{N^{2}} \sum_{k=1}^{n}\left|a_{j, k}\right|^{2} \min \left\{\frac{n}{3}, \frac{1}{1-\beta}\right\}\right)^{3 / 2} .
\end{aligned}
$$

We note that (2.15) implies that $\left|G_{3}(Z)\right|$ is bounded by $\sqrt{3}(\gamma(1 / 3))^{3 / 2}$, which is however of worse order.

The following result is a consequence of Theorem 2.8, (2.14) and (2.15).

Corollary 2.12. If $\gamma<1$, then

$$
\begin{aligned}
\left|\frac{(N-n) !}{N !} \operatorname{Per}(Z)-\prod_{k=1}^{n} \widetilde{z}_{k}\right| \leq & \gamma(1 / 2)+\frac{3^{1 / 4} C_{3} \gamma^{3 / 2}}{(1-\gamma)^{3 / 4}} \\
\left|\frac{(N-n) !}{N !} \operatorname{Per}(Z)-H_{2}(Z)\right| \leq & \sqrt{3} \sum_{j=1}^{N}\left(\frac{1}{N^{2}} \sum_{k=1}^{n}\left|a_{j, k}\right|^{2} \min \left\{\frac{n}{3}, \frac{1}{1-\beta}\right\}\right)^{3 / 2} \\
& +\frac{2^{1 / 2} C_{4} \gamma^{2}}{(1-\gamma)^{3 / 4}}
\end{aligned}
$$

where the second inequality requires $n \geq 2$.

Proof of Theorem 1.1. Inequality (1.6) follows from (2.16) and (1.4), while (1.7) can be easily shown using (2.17), (2.13) and the representation

$$
\begin{aligned}
\int f \mathrm{~d}\left(P^{Y_{n}}-Q_{2}\right) & =\int\left(\frac{(N-n) !}{N !} \sum_{j \in \underline{N}_{\neq}^{n}} \prod_{k=1}^{n} f_{k}\left(X_{j_{k}}(\omega)\right)-\prod_{k=1}^{n} \zeta_{k}(\omega)\right. \\
& \left.+\frac{1}{N(N-1)} \sum_{K \subseteq \underline{n}:|K|=2} \sum_{j=1}^{N} \prod_{k \in K}\left(f_{k}\left(X_{j}(\omega)\right)-\zeta_{k}(\omega)\right) \prod_{k \in \underline{n} \backslash K} \zeta_{k}(\omega)\right) \mathrm{d} P(\omega)
\end{aligned}
$$

for $f=\bigotimes_{k=1}^{n} f_{k} \in \mathcal{F}_{n}$, where $\zeta_{k}(\omega)=\frac{1}{N} \sum_{j=1}^{N} f_{k}\left(X_{j}(\omega)\right)$.

We now show that the singularity in (2.7) can be removed.

Theorem 2.13. For fixed $\ell \in \underline{n}$, let $\kappa_{\ell}$ be the smallest absolute constant such that, without any restrictions on $\gamma$,

$$
\left|\frac{(N-n) !}{N !} \operatorname{Per}(Z)-H_{\ell}(Z)\right| \leq \kappa_{\ell} \gamma^{(\ell+1) / 2} .
$$

Then $\kappa_{\ell} \leq \frac{(\ell+1)^{1 / 4} C_{\ell+1}}{\left(1-x_{\ell}\right)^{3 / 4}}$, where $x_{\ell} \in(0,1)$ is the unique positive solution of the equation

$$
2+2^{1 / 4} C_{2} x\left(\frac{1-x^{\ell-1}}{1-x}\right)^{3 / 4}=(\ell+1)^{1 / 4} C_{\ell+1} \frac{x^{(\ell+1) / 2}}{(1-x)^{3 / 4}}, \quad(x \in(0,1)) .
$$

In particular, $\kappa_{1} \leq 3.57, \kappa_{2} \leq 5.53$ and $\kappa_{3} \leq 7.08$. 
Proof. Dividing (2.18) by $x^{(\ell+1) / 2}$ yields a decreasing left-hand side, whereas the right-hand side remains increasing in $x$. Therefore (2.18) has indeed a unique positive solution $x_{\ell} \in(0,1)$. Similarly as in the proof of Theorem 2.8, we have

$$
\begin{aligned}
& \left|\frac{(N-n) !}{N !} \operatorname{Per}(Z)-H_{\ell}(Z)\right| \leq \frac{(N-n) !}{N !}|\operatorname{Per}(Z)|+\left|H_{\ell}(Z)\right| \leq 2+\sum_{m=2}^{\ell}\left|G_{m}(Z)\right| \\
& \leq 2+2^{1 / 4} C_{2} \gamma\left(\sum_{m=0}^{\ell-2} \sqrt{m+1} \gamma^{m}\right)^{1 / 2} \leq 2+2^{1 / 4} C_{2} \gamma\left(\frac{1-\gamma^{\ell-1}}{1-\gamma}\right)^{3 / 4}=: h(\gamma) .
\end{aligned}
$$

If $\gamma \in\left[0, x_{\ell}\right]$, we obtain by Theorem 2.8 that

$$
\left|\frac{(N-n) !}{N !} \operatorname{Per}(Z)-H_{\ell}(Z)\right| \leq(\ell+1)^{1 / 4} C_{\ell+1} \frac{\gamma^{(\ell+1) / 2}}{(1-\gamma)^{3 / 4}} \leq \frac{(\ell+1)^{1 / 4} C_{\ell+1}}{\left(1-x_{\ell}\right)^{3 / 4}} \gamma^{(\ell+1) / 2} .
$$

If $\gamma \in\left(x_{\ell}, \infty\right)$, then

$$
\left|\frac{(N-n) !}{N !} \operatorname{Per}(Z)-H_{\ell}(Z)\right| \leq h(\gamma) \leq \frac{h\left(x_{\ell}\right)}{x_{\ell}^{(\ell+1) / 2}} \gamma^{(\ell+1) / 2}=\frac{(\ell+1)^{1 / 4} C_{\ell+1}}{\left(1-x_{\ell}\right)^{3 / 4}} \gamma^{(\ell+1) / 2} .
$$

It remains to use that $x_{1} \leq 0.5611, x_{2} \leq 0.7222$ and $x_{3} \leq 0.7812$.

\section{ACKNOWLEDGMENTS}

The author is indebted to an anonymous reviewer for bringing to his attention the result given in Lemma 2.1 and for the indication of how it helps to improve the previous version of Lemma 2.2. This led to the significant improvement of constants in several upper bounds. The author also thanks Lutz Mattner for helpful comments.

\section{REFERENCES}

[1] S. Banach, Über homogene Polynome in $\left(L^{2}\right)$, Studia Math. 7 (1938), 36-44.

[2] Sergey G. Bobkov, Concentration of normalized sums and a central limit theorem for noncorrelated random variables, Ann. Probab. 32 (2004), no. 4, 2884-2907, DOI 10.1214/009117904000000720. MR2094433 (2005i:60041)

[3] Sergey G. Bobkov, Generalized symmetric polynomials and an approximate de Finetti representation, J. Theoret. Probab. 18 (2005), no. 2, 399-412, DOI 10.1007/s10959-005-3509-6. MR.2137450(2006c:28003)

[4] Eric Carlen, Elliott H. Lieb, and Michael Loss, An inequality of Hadamard type for permanents, Methods Appl. Anal. 13 (2006), no. 1, 1-17, DOI 10.4310/MAA.2006.v13.n1.a1. MR:2275869(2007k:15013)

[5] Gi-Sang Cheon and Ian M. Wanless, An update on Minc's survey of open problems involving permanents, Linear Algebra Appl. 403 (2005), 314-342, DOI 10.1016/j.laa.2005.02.030. MR2140290 (2006b:15012)

[6] P. Diaconis and D. Freedman, Finite exchangeable sequences, Ann. Probab. 8 (1980), no. 4, 745-764. MR0577313 (81m:60032)

[7] Seán Dineen, Complex analysis on infinite-dimensional spaces, Springer Monographs in Mathematics, Springer-Verlag London Ltd., London, 1999. MR1705327 (2001a:46043)

[8] G. H. Hardy, J. E. Littlewood, and G. Pólya, Inequalities, Cambridge, at the University Press, 1952. 2d ed. MR0046395 (13,727e)

[9] Lawrence A. Harris, Bernsteı̆n's polynomial inequalities and functional analysis, Irish Math. Soc. Bull. 36 (1996), 19-33. MR.1387033 (97h:46070)

[10] Edwin Hewitt and Leonard J. Savage, Symmetric measures on Cartesian products, Trans. Amer. Math. Soc. 80 (1955), 470-501. MR0076206 (17,863g)

[11] Lars Hörmander, On a theorem of Grace, Math. Scand. 2 (1954), 55-64. MR0062844(16,27b) 
[12] O. D. Kellogg, On bounded polynomials in several variables, Math. Z. 27 (1928), no. 1, 55-64, DOI 10.1007/BF01171085. MR:1544896

[13] Henryk Minc, Permanents, Encyclopedia of Mathematics and its Applications, vol. 9999, Addison-Wesley Publishing Co., Reading, Mass., 1978. With a foreword by Marvin Marcus. MR0504978 (80d:15009)

[14] D. S. Mitrinović, Analytic inequalities, Springer-Verlag, New York, 1970. In cooperation with P. M. Vasić. Die Grundlehren der mathematischen Wissenschaften, Band 165. MR0274686 (43 \#448)

[15] B. Roos, Binomial approximation to the Poisson binomial distribution: the Krawtchouk expansion (English, with Russian summary), Teor. Veroyatnost. i Primenen. 45 (2000), no. 2, 328-344, DOI 10.1137/S0040585X9797821X; English transl., Theory Probab. Appl. 45 (2001), no. 2, 258-272. MR 1967760 (2003k:60099)

[16] B. Roos, Multinomial and Krawtchouk approximations to the generalized multinomial distribution (English, with Russian summary), Teor. Veroyatnost. i Primenen. 46 (2001), no. 1, 117-133, DOI 10.1137/S0040585X97978750; English transl., Theory Probab. Appl. 46 (2002), no. 1, 103-117. MR1968708(2004b:62043)

FB IV - Department of Mathematics, University of Trier, 54286 Trier, Germany

E-mail address: bero.roos@uni-trier.de 